\title{
EL FONEMA FRICATIVO / S / EN EL HABLA CULTA DE SAN JOSÉ: IMPLICACIONES TEÓRICAS EN EL SISTEMA FONOLÓGICO DE LAS CORONALES
}

\author{
Annette Calvo Shadid
}

\begin{abstract}
RESUMEN
El presente estudio ofrece un análisis cuantitativo de las variantes del segmento / s / en el habla culta de San José. Con base en los datos anteriores, la autora propone que el segmento / s / ha dejado de ser un fonema predorso alveolar para transfonologizarse en uno ápico o predorso dental por la presión estructural que ejerce sobre este el segmento $/ \mathrm{r} /$ (el cual se ha transfonologizado en una variante fricativa retrofleja). De este modo, la autora supone que los segmentos fricativos coronales, a saber, / s / (ápico o predorso dental) y / $t$ / (retroflejo) se distribuyen en una serie organizada de fricativas coronales, en la cual/s / es anterior y / $\{/$, posterior.
\end{abstract}

\begin{abstract}
This article presents a quantitative analysis of the variants of the / $/$ / segment that appear in the standard speech of San Jose. Based on this data, the author proposes that the / s / segment is not a predorsal alveolar phoneme anymore since it has been transphonologized into another with apical or predorso-dental features, due to structural the pressure from the / $\mathrm{r} /$ segment (transphonologized into a retroflex fricative). The author claims that fricative coronal segments / s / (apical or predorsal dental) and / $\mathrm{t} /$ (retroflex) are distributed in an organized series of coronal fricatives where $/ \mathrm{s} /$ is anterior and $/ \mathrm{f} /$ is posterior.
\end{abstract}

\section{Introducción}

\subsection{Sobre los segmentos coronales}

De acuerdo con Chomsky y Halle (1968: 174), los segmentos coronales se producen con la parte predorsal de la lengua elevada con respecto a su posición neutral; los sonidos no coronales se producen con esta parte de la lengua en posición neutral. Los autores consideran que los sonidos coronales son, entonces, aquellos que se producen en la región dental, alveolar y palatoalveolar.

Estudios posteriores ${ }^{1}$ han redefinido el concepto de coronal, como la serie de segmentos que se producen con la lámina, incluyendo en esta la punta de la lengua. De acuerdo con Keating (1991: 31) la lámina o blade se extiende de 1 a 2 cms. detrás de la punta de la lengua; es por esto que los segmentos coronales pueden ser apicales, laminales o ápicolaminales. 
De acuerdo con la redefinición de coronal, actualmente se reconocen como tales los puntos de articulación dental, alveolar, palatoalveolar, retroflejo y palatal ${ }^{2}$. Ladefoged y Maddieson (1986) agregan dos puntos de articulación menos comunes, a saber, linguolabial e interdental.

Se ha observado ${ }^{3}$ que las coronales son las consonantes más frecuentes en las lenguas y si una lengua solamente posee una consonante fricativa, esta será coronal, a saber, $/ \mathrm{s} /{ }^{4}$. Por otro lado, estos sonidos incluyen más contrastes de punto y de modo que otras clases de consonantes y, además, estudios de adquisición han demostrado que las coronales, junto con las labiales, son las primeras consonantes adquiridas por los niños ${ }^{5}$. Por estas razones, se han considerado como las consonantes menos marcadas.

Paradis y Prunet (1991: 8-12) presentan una enumeración de algunas propiedades de las coronales, la cual se presenta a continuación.

La primera de tales propiedades es la asimilación. Las coronales son mucho más asimilables en su punto de articulación que otro tipo de consonantes ${ }^{6}$. Por ejemplo, en castellano y en catalán, la nasal coronal / $\mathrm{n} /$ se asimila a todas las consonantes, en tanto que otras nasales no coronales, tales como / $\mathrm{n} /$ en español, y / n / y / $\mathrm{g} /$ en catalán, no se asimilan. Únicamente se asimila / $\mathrm{m}$ / en labiodental / $\mathrm{m}$ / en ambas lenguas.

Por otro lado, las coronales también son consonantes que asimilan otros segmentos. Por ejemplo, es frecuente que la nasalidad de una / n / que anteceda a una vocal, la nasalice. Otro caso se da en el español de Costa $\mathrm{Rica}^{7}$ en los grupos consonánticos str y rs, cuyos segmentos son todos coronales. Estos se suelen fundir en una variante ápico alveolar asibilada sorda [ $\mathrm{S}$ ], en los casos de almuerzo e instrumento, entre otros.

La neutralización es otra de las propiedades características de las coronales. Algunos estudios citados por Paradis y Prunet (1991: 9) demuestran que, en algunas lenguas, ciertos segmentos no coronales se neutralizan en coronales en posición de coda ${ }^{8}$. Parece que en esta posición se favorece la aparición de estos segmentos, los cuales se consideran más simples.

Las coronales se caracterizan, además, por su transparencia. En otros términos, los segmentos transparentes permiten que otro segmento se extienda a través de ellos. Un ejemplo de esto lo ofrecen los mismos autores con el fula. En esta lengua, las vocales pueden extenderse a través de segmentos coronales, pero no a través de otros que no lo sean'.

Por último, las coronales se caracterizan por su frecuencia: como se mencionó anteriormente, se afirma que las coronales son las consonantes más frecuentes. Paradis y Prunet (1991: 11) proponen algunas distinciones entre los diferentes tipos de frecuencia, a saber, frecuencia de inventario: por ejemplo, en inglés existen trece coronales, cinco labiales y dos velares; el francés tiene nueve coronales, cinco labiales y dos velares. Por otro lado, son las consonantes más frecuentes en el alfabeto fonético internacional. En cuanto a la frecuencia de aparición, se ha observado que las coronales son las consonantes más frecuentes en la conversación ${ }^{10}$.

Las propiedades anteriores le otorgan a las coronales un estatus especial dentro de las consonantes. En este estudio se tratará de observar, mediante un análisis de variación, las particularidades del segmento / s / y sus implicaciones en el sistema de las fricativas coronales en el habla culta de San José.

Es importante señalar que, entre las coronales, el segmento / s / se caracteriza, de acuerdo con Chomsky y Halle, por ser estridente ${ }^{11}$. Este rasgo se restringe solo a las consonantes 
fricativas y africadas, y es definido (Chomsky y Halle 1968/79: 226) como una cierta turbulencia generada cuando la corriente de aire pasa sobre una superficie, dependiendo de la naturaleza de esta. El aumento de incidencia se ve favorecido por una superficie dura, por la mayor rapidez de la corriente y por la proximidad del ángulo de incidencia a los noventa grados.

Ladefoged (1982) presenta un sistema ternario alternativo a la teoría de los rasgos binarios de Chomsky y Halle. Por el modo de articulación, divide los segmentos en oclusivos, fricativos y aproximantes. Chomsky y Halle no reconocen la fricción como una propiedad fonética, pues de este modo resultarían redundantes algunos de sus rasgos distintivos ${ }^{12}$. Además, esta clasificación ternaria muestra que el proceso de cambio de una oclusiva a una fricativa es un debilitamiento articulatorio similar al proceso de cambio de una fricativa a una aproximante, lo cual no se permite mostrar formalmente por medio de rasgos binarios.

Ladefoged y Maddieson (1986) cuestionan el rasgo estridente de Chomsky y Halle. Encuentran que es más apropiado, por razones fonológicas y fonéticas, la división de las consonantes fricativas en sibilantes y no sibilantes.

A este respecto proponen (1986: 92) que la clasificación fonológica de las fricativas debe requerir, además, el rasgo sibilante. La diferencia entre las consonantes sibilantes y las no sibilantes se da dependiendo de si en la constricción se produce un chorro de aire que topa con un obstáculo formado, por ejemplo, con los dientes. Las no sibilantes se forman mediante una turbulencia generada en la constricción misma.

Los autores señalan que las fricativas más usuales en la región alveolar son las sibilantes $s$ y $z$. En ellas, la principal fuente del sonido es la turbulencia producida cuando el chorro de aire producido por la constricción alveolar toca los dientes y forma un obstáculo que sigue a la constricción misma.

Ladefoged y Madieson presentan una nueva descripción general de las fricativas basada en puntos de articulación y en la clasificación entre sibilantes y no sibilantes, como se describe en la Tabla 1.

Tabla 1

Clasificación de fricativas en centrales y no centrales

(1) Región no central

labio- linguo- inter- | | bilabial dental labial dental I | palatal velar uvular faríngea epiglotal

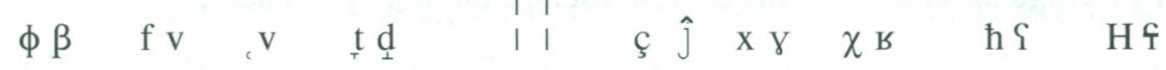

(2) Región central, sibilantes y no sibilantes

\begin{tabular}{|c|c|c|c|c|c|c|}
\hline ental & alveolar & $\begin{array}{c}\text { postalveolar } \\
\text { plana } \\
\text { (retrofl.) }\end{array}$ & $\begin{array}{c}\text { postalveolar } \\
\text { cóncava } \\
\text { (palatoalv.) }\end{array}$ & $\begin{array}{l}\text { postalveolar } \\
\text { palatalizada } \\
\text { (alveopala.) }\end{array}$ & $\begin{array}{l}\text { postalveolar } \\
\text { cerrada }\end{array}$ & $\begin{array}{c}\text { sublaminal } \\
\text { palatal } \\
\text { (retrofl.) }\end{array}$ \\
\hline
\end{tabular}

no sib. $\quad \theta \partial \quad, \theta, \partial$

sibil. S S Z S Z

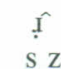

$\int 3$

$6 \mathrm{Z}$

$\widehat{\mathbf{S}} \hat{\mathbf{Z}}$

$\mathrm{S}$ 
De acuerdo con esta tabla, los sonidos se dividen en fricativos localizados en la región no central y en fricativos que se localizan en la región central.

La región no central abarca los puntos de articulación que no están en la parte central de la cavidad oral; se considera que en esta área se localizan los sonidos bilabiales, labiodentales, linguolabiales, interdentales (anteriores), y luego, los palatales, velares, uvulares, faringales y epiglotales (posteriores). Todas estas fricativas son no sibilantes.

En la región central se ubican los sonidos dentales, alveolares, postalveolares (también retroflejos) y palatales retroflejos. En esta área se localizan todos los sonidos sibilantes y también algunos no sibilantes.

Como se puede observar, las clasificaciones articulatorias de Ladefoged (1982) y Ladefoged y Maddieson (1986) presentan rasgos multivalentes, lo cual se acerca más a la clasificación tradicional propuesta por la International Phonetic Association (IPA).

Esta clasificación de sonidos fricativos sibilantes y no sibilantes resulta más acorde con esta investigación, por razones teóricas y articulatorias.

\subsection{La variación de / s / en diferentes dialectos del español de América: estudios previos}

El segmento / s / es muy susceptible de variación y, por tariı, el más estudiado de todos en el mundo hispánico, según López Morales (1989: 86) ${ }^{13}$.

En posición intervocálica se han registrado tanto variantes sordas más o menos anteriorizadas como sus correspondientes sonoras ${ }^{14}$, pero ha sidə sobre todo en posición implosiva, en donde se han registrado la mayor cantidad de variantes.

Entre las variantes registradas en este contexto se encuentran desde realizaciones sibilantes apicales, predorso alveolares, predorso dental $ڤ s$ y palatales hasta la elisión, pasando por las aspiradas y velarizadas con diversos grados de sonoridad, asimiladas a la consonante siguiente, variantes bilabiales y labiodentales ante bilarial sonora, hasta realizarse como vibrante simple fricativa [ r ]. El segmento / s / en posición inicial de sílaba (no intervocálica) ha sido poco estudiado y no se dispone de estudios de variación. En los textos tradicionales de ortología y fonética y fonología españolas, las variantes reconocidas son la apicoalveolar, la cual es mucho más extendida en España que en América, y la predorsal, con variantes en diferentes regiones, registrada en Andalucía y América. Canfield (1988: 23) menciona una variante dental frecuente en El Salvador, Honduras, Nicaragua y en algunas áreas costeras de Venezuela, Colombia y Ecuador.

\subsection{La variación de / s / en el español de Costa Rica}

Chavarría Aguilar (1951: 250) registra una variante alveolar groove spirant generalmente sorda, pero que se sonoriza ante consonante sonora. Agüero (1962: 27) caracteriza el segmento / s / como sigue:

Nuestra es la predorsal convexa de América y Andalucía. En la Meseta Central y otros lugares excepto en Guanacaste y parte de Puntarenas- jamás se aspira. Solamente se oye a veces en nojotros (nosotros) y lojotros (los otros), pero entre campesinos. 
Resnick (1975: 205) reconoce una variante apicoalveolar o apicodental en el Valle Central, la cual considera predominante, y otra predorsal o dorsoalveolar convexa de uso general. Ambas son sibilantes. Considera ocasional el uso de una apicodental plana no sibilante.

Algunos autores han considerado que variantes similares a esta última son ceceantes, y se han registrado también en otros países como Argentina, Bolivia, Colombia, Ecuador, Honduras, México, Nicaragua y Puerto Rico ${ }^{15}$. De acuerdo con esto, las variedades del español de América en que se registran serían ceceantes, como citan Zamora Munné y Guitart (1982: 90):

\footnotetext{
En ciertos dialectos americanos, (...) se registra el fenómeno denominado ceceo (...). El fenómeno es mucho más general en Andalucía. (...) Como ha observado Navarro Tomás, la articulación ceceante puede ser lo mismo dental que interdental, pero lo que es común a todas las realizaciones es la forma de la estrechez creada por lengua y dientes, que es hendida como la de / $\theta$ / y no acanalada como la de / $/$ /.
}

Resnick (1975:212) cita también para Costa Rica variantes sonoras en posición intervocálica interna de palabra, lo cual es un rasgo característico del habla serrana de Ecuador. Su uso en Costa Rica se ha registrado esporádicamente en la clase media. En esta investigación, la autora ha encontrado el uso de esta variante muy frecuentemente ante consonante sonora y, en posición intervocálica, con relativa frecuencia.

\title{
1.4. Objetivo de la investigación
}

De acuerdo con todo lo anterior, se puede observar que el segmento / s / ha sido estudiado en numerosas ocasiones en el mundo hispánico. En la investigación presente se plantea la variación de este segmento en el habla culta de San José. Para esto, se analiza la primera generación tanto femenina como masculina de la muestra tomada de los materiales para el estudio del habla culta de esta ciudad. Corresponde a las cuatro informantes femeninas y a los cuatro informantes masculinos; esto es, la primera generación completa.

Posteriormente se procederá a determinar cuantitativamente las variantes del segmento en cuestión, y luego, se confirmará que, así como el segmento / $\mathrm{r}$ / se ha transfonologizado en una variante fricativa o aproximante retrofleja, es decir, en una coronal no anterior, del mismo modo / $\mathrm{s}$ I, por presión estructural, ha dejado de ser un fonema predorso alveolar para transfonologizarse, a su vez, en uno ápico o predorso dental ${ }^{16}$; ambos segmentos son coronales anteriores.

De este modo, se pretende aquí confirmar que los segmentos fricativos son coronales, a saber, / s / (ápico o predorso dental), y / £ /, (retroflejo), se distribuyen en una serie organizada de fricativas coronales, en la cual / $\mathrm{s} / \mathrm{es}$ anterior $\mathrm{y} / \mathrm{f} /$, posterior.

\subsection{Los contextos y las variantes}

Se tomaron en cuenta los siguientes contextos en posiciones inicial y final de sílaba, a saber:

\author{
- Ante vocal \\ - Entre consonante y vocal \\ - En posición inicial de enunciado
}


- Entre vocal y consonante

- Ante pausa

Las variantes registradas fueron las siguientes, de la más frecuente a la de menor frecuencia, en un total de 618 sonidos registrados:

$[\underset{n}{\mathrm{~s}}]=$ fricativa sibilante dental sorda

$[\mathrm{s}]=$ fricativa sibilante predorso alveolar sorda

$[ð]=$ fricativa dental sonora

$[\mathrm{z}]=$ fricativa sibilante alveolar sonora

$[\mathrm{h}]=$ aproximante faríngea sorda (/ s / aspirada)

\section{Presentación de los resultados}

En términos generales, la variante más frecuente es [ $\mathbf{s}$ ] en los dos grupos estudiados, a saber, la fricativa sibilante dental sorda, con un porcentaje del 49,2\%. En segundo término sigue [ s ], con un 42,32\%; luego [ J ], con un 4,46\%; [ z ] con 2,75\% y, por último, [ h ], con 1,27\%.

Tabla 2

/ s / en informantes masculinos y femeninos de la I generación (términos porcentuales)

$\begin{array}{lcccccc}\text { CONTEXTOS } & {[\mathbf{s}]} & {[\mathbf{s}]} & {[\mathbf{z}]} & {[\mathbf{\delta}]} & \text { [ h ] } & \text { TOTAL } \\ \text { V-V/V\#_V } & 54,21 & 36,95 & 5,62 & - & 3,21 & 100 \\ \text { C_V/C\#_V } & 43,28 & 55,97 & 0,75 & - & - & 100 \\ \text { V_C } & 39,50 & 35,29 & 1,68 & 23,53 & - & 100 \\ \text { V_\#\# } & 55,05 & 44,95 & - & - & - & 100 \\ \text { \#\#_V } & 54,28 & 45,72 & - & - & - & 100\end{array}$

En primer lugar, la posición intervocálica en palabras como efiçiente, cas $a$, etc., presenta un porcentaje mucho mayor de la variante dental sorda que los demás contextos. Es importante señalar aquí el uso de la variante sonora en posición intervocálica, la cual había sido registrada en otros países de América, principalmente en Ecuador, y en Costa Rica, fue registrada por Resnick (1975) (ver arriba). La investigadora ha percibido este rasgo con mucha mayor frecuencia en los medios de comunicación, en periodistas, locutores y, en general, en personas de la clase media de San José.

Por otro lado, la aspiración se da en un porcentaje muy bajo, y solamente en posición inicial de sílaba y entre vocales.

El siguiente contexto analizado, / s / entre consonante y vocal, en palabras como abs $o$ luto, marzo, etc., mostró una mayor frecuencia de variantes alveolares, si bien es un hecho que la variante dental es también muy frecuente en este contexto. Se observa aquí que, en todo caso, la posición intervocálica presenta una frecuencia bastante mayor del uso de esta variante. 
El contexto de / s / entre vocal y consonante se observó en palabras como esperar, os $c u$ ro, mismo, esbelta, etc. En este contexto, se observó una mayor preferencia por la variante dental, al igual que en posición intervocálica, aunque también se usa con frecuencia la variante alveolar.

Es importante observar aquí el uso de las variantes sonoras, en total $25,21 \%$, por asimilación con la consonante siguiente si esta era sonora, en casos como mismo, es bello, desde, etc. Es interesante señalar que, así como / s / en estos casos se sonoriza, también pierde su sibilancia. Los casos de [ z ], es decir, de la sibilante sonora representan solamente un 1,68\%, mientras que el porcentaje de la fricativa o aproximante sonora no sibilante, [ $\succsim$ ], es mucho mayor. Una posible explicación para la desasibilación de [ s ] en este contexto es el debilitamiento o el relajamiento que sufren los segmentos en posición posnuclear de sílaba.

Además de sonoro y no sibilante, la variante [ ð ] es también dental. Por lo tanto, el porcentaje de variantes dentales en este contexto es mucho mayor; en total, 63,03\%. Si lo comparamos con el de las variantes alveolares, en total, 36,97\%, la diferencia entre ambos tipos de variantes es bastante significativa. Es decir, que es en este contexto en el cual aparecen más frecuentemente las variantes dentales, en general.

En los contextos posición final absoluta y posición inicial de enunciado, los resultados son similares: una frecuencia mayor de variantes dentales y, en un poco menor proporción, variantes alveolares. No se observaron otras variantes.

Tabla 3

Variación de / s / distribuido por variable sexo I generación (términos porcentuales)

\begin{tabular}{|c|c|c|c|c|c|c|c|c|c|c|}
\hline VARIA & & & & & & & & & & 1] \\
\hline SEXO & $\mathrm{M}$ & F & M & F & M & F & M & $\mathrm{F}$ & M & $\mathrm{F}$ \\
\hline$V_{-} \mathrm{V}$ & 60 & 49,26 & 30,44 & 42,54 & 9,56 & 2,23 & - & - & - & 5,97 \\
\hline $\mathrm{C}_{-} \mathrm{V}$ & 36,84 & 48 & 61,41 & 52 & 1,75 & - & - & - & - & - \\
\hline $\mathrm{V}_{-} \mathrm{C}$ & 43,18 & 37,34 & 34,10 & 36 & - & 2,66 & 22,72 & 24 & - & - \\
\hline V_\#\# & 41,94 & 62,07 & 58,06 & 37,93 & & - & - & - & - & - \\
\hline \#\#_V & 46,66 & 60 & 53,34 & 40 & - & - & - & - & - & - \\
\hline
\end{tabular}

Los resultados por la variable sexo no permiten establecer diferencias significativas. La aspiración se observó en pocos casos en las mujeres, y en ningún caso en los hombres. Sin embargo, esto no indica que los hombres no aspiren esporádicamente, tanto como las mujeres, en posición inicial de sílaba, especialmente entre vocales.

Por otro lado, se observa que los hombres realizan con más frecuencia la variante sonora ante vocal, pero la diferencia en relación con las mujeres no es tan grande como para afirmar que ellos sonoricen más en dicho contexto.

\section{Conclusiones}

Los resultados de esta investigación permiten demostrar, cuantitativamente, que las variantes dentales son más frecuentes que las alveolares en el habla culta de San José. 
Lo anterior confirma la hipótesis de que, así como el segmento / r / se ha transfonologizado en una variante fricativa o aproximante retrofleja, es decir, en una coronal no anterior, del mismo modo / s /, por presión estructural, ha dejado de ser un fonema predorso alveolar para transfonologizarse, a su vez, en uno ápico o predorso dental. Ambos son segmentos coronales anteriores.

De este modo, la serie fonemas fricativos en el habla culta de San José, presenta la siguiente distribución:

\begin{tabular}{|c|c|c|c|}
\hline \multicolumn{4}{|c|}{$\begin{array}{l}\text { Distribución de los fonemas fricativos } \\
\text { en el habla culta de San José }\end{array}$} \\
\hline Labiales & & & Dorsales \\
\hline 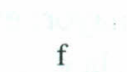 & $\underset{s}{\text { Anter. }}$ & No anter. & \\
\hline
\end{tabular}

\section{Notas}

1. Cf. Halle y Stevens (1979), Keating (1988, 1991).

2. El punto de articulación palatal ha sido considerado coronal, de acuerdo con criterios fonológicos y fonéticos. Para esto, cf. Keating (1991). Desde 1979, Halle y Stevens proponen una redefinición de coronal, tal que incluya las palatales, en el sentido de que las coronales se producen con el predorso o mediodorso de la lengua, the blade or the front of the tongue.

3. Cf. Paradis y Prunet (1991: 1 y ss.)

4. Cf. Maddieson (1987: 31) (citado por Paradis y Prunet).

5. Cf. Stoel-Gammon (1985) y Vihman y otros (1986) (citados por Paradis y Prunet).

6. Cf. Kiparky (1985), Avery y Rice (1988, 1989 a, b) (citados por Paradis y Prunet).

7. Cf. Calvo Shadid (1995) para observar la asimilación de estos grupos consonánticos en una variante ápico alveolar asibilada sorda [ s ]. Cf., además, Sánchez Corrales (1985).

8. Paradis y Prunet citan a Cho (1988) para el coreano, a Cook (1976) para el carrierés, lengua atapasca, y a Paradis (1986), para el fula.

9. Cf. Paradis y Prunet (1989). En fula existe extensión vocálica a través de la coronal / $\mathrm{t} / \mathrm{para}$ formar los paradigmas de imperfecto, y a través de la coronal / r / para los marcadores nominales.Sin embargo, esta extensión se bloquea si aparece otro tipo de consonantes.

10. Paradis y Prunet citan los estudios de Fry (1947), quien demuestra que las consonantes más frecuentes en la conversación en inglés británico del sur son, en orden descendente, las coronales $n, t, d, s$ y $l$.

11. Rasgo utilizado primeramente por Jakobson y Halle (1956).

12. Cfr. Ladefoged y Maddieson (1986: 91-2).

13. Cfr., entre otros, Zamora Vicente (1970: 416-17), Cuestionario... (1973: 44-49), Resnick (1975: 36-7); Fontanella de Weinberg (1967, 1973, 1974 a y b), Sanicky (1982/83) y Donni de Mirande (1987) para Argentina; Montes Giraldo (1984) para Colombia; Terrel (1975 a y b, 1978a y 1979) para Cuba; Hammond (1989) para la variedad cubana de Miami; Lipsky (1989) para Ecuador; Lipsky (1986) para Honduras; Avi- 
la (1973) y López Chaves (1977) para México; Cedergreen (1973 y 1978) para Panamá; Caravedo (1983) y Hundley (1983) para Perú; Beym (1963) y Terrel (1978 c) para el español porteño; Alemán (1977), Terrel (1978 a y b), Alba (1982 a), Hammond (1982), López Morales (1983) y Hochberg (1986) para Puerto Rico; Poplack (1979) para la variedad puertorriqueña de Filadelfia; Núñez Cedeño (1980), Alba (1982 b) y Terrel (1986) para República Dominicana; Longmire (1976) para Venezuela.

14. Cfr. Cuestionario... (1973: 44) y, entre otros, Resnick (1975: 212), Lipsky (1989), Zamora Muné y Guitart (1982:97) para la sonorización de /s/ en este contexto en el Ecuador. También en Resnick (1975: 212) y en Flórez (1964) se ha registrado su uso esporádico en Colombia y Costa Rica.

15. Cfr., entre otros, Canfield (1953 y 1962), Zamora Vicente (1970: 417-18), Resnick (1975: 210-11).

16. Cfr. las citas de Resnick y Navarro Tomás en el segmento sobre los estudios acerca del español de Costa Rica. Como se puede observar, ambos investigadores confirman el uso de variantes dentales en el español de Costa Rica y de otras partes de América.

\section{Bibliografía}

Alba, O. 1982a. Estratificación social del español de Santiago de los Caballeros. La / s / implosiva. Tesis de maestría. Río Piedras: Universidad de Puerto Rico.

1982b. "Función del acento en el proceso de elisión de la / s / en la República Dominicana". En: Alba (ed.).

Alemán, I. 1977. S final de sílaba implosiva y de palabra en el español de Puerto Rico. Tesis de maestría. Río Piedras: Universidad de Puerto Rico.

Avery, P. y K. Rice. 1988. "Underspecification theory and coronal node". Working papers in linguistics. 9:101-19.

1989a. "Segment structure and coronal underspecification". Phonology. 6:170-200.

Ávila, R. 1973. "Realizaciones tensas de / s / en la ciudad de México". Anuario de Letras. 11: 235-9.

Calvo Shadid, A. 1995. "Variación fonética de / s / y / r / en el habla culta de San José". Revista de Filología y Lingüística de la Universidad de Costa Rica. 1: 115-34.

Canfield, D. L. 1962. La pronunciación del español en América. Ensayo histórico-descriptivo. Bogotá: Instituto Caro y Cuervo.

1982. Spanish pronunciation in the Americas. Chicago: University Press. Versión española, D. L. Canfield (1988), El español de América: fonética.

Caravedo, R. 1983. Estudios sobre el español de Lima. Variación contextual de la sibilante. Lima: Pontificia Universidad Católica del Perú. 
Cedergren, H. 1973. The interplay of social and linguistic factors in Panamá. Tesis doctoral: Universidad de Cornell.

1978. "En torno a la variación de $s$ final en Panamá: análisis cuantitativo". En: H. L. Morales (ed.).

Cho, Y. 1988. Korean assimilation. WCCFL. 7:1-52.

Chomsky, N. y M. Halle. 1968. The sound patterns of English. New York: Harper \& Row. Versión española: Chomsky \& Halle (1979): Principios de fonología generativa. Madrid: Fundamentos.

Comisión de Lingüística Iberoamericana (P.I.L.E.I.). 1973. Cuestionario para el estudio coordinado de la norma lingüística culta de las principales ciudades de Iberoamérica y de la Península Ibérica. I Fonética y Fonología. Madrid: Consejo Superior de Investigaciones Científicas.

Donni de Mirande, N. E. 1984. "Un cambio lingüístico en Rosario. A propósito de las realizaciones de / s /". Cahiers de Lexicologie. 3: 117-42.

Flórez, L. 1964. "El español hablado en Santander". Anuario de Letras. 4: 71-94.

Fontanella de Weinberg, M. B. 1967. "La s postapical en el español bonaerense". Thesaurus. 27: 394-400.

1973. "Comportamiento ante -s de hablantes femeninos y masculinos del español bonaerense". Romance Philology. 27: 50-8.

1974a. Análisis sociolingüístico de un aspecto del español bonaerense. La -s en Bahía Blanca. Bahía Blanca: Universidad Nacional del Sur.

1974b. “Aspectos sociolinguíisticos del uso de -s en el español bonaerense”. Orbis. 23: 85-98.

Halle, M. y K. N. Stevens. 1979. "Some Reflections on the Theoretical Bases of Phonetics". En: B. Lindblom y S. Ohman (eds.), Frontiers of Speech Communication Research. Londres: Academic Press.

Hammond, R. M. 1980. "Las realizaciones fonéticas del fonema / s / en el español cubano rápido de Miami”. En: G. E. Scavnicky (ed.). 1980. Dialectología hispanoamericana. Washington: Georgetown University Press.

1989. "El fonema / s / en el español jíbaro. Cuestiones teóricas". En O. Alba (ed.). 
Hochberg, J. G. 1986. "El fonema / s / en el español jíbaro. Cuestiones teóricas". Language. 62: 609-21.

Hundley, J. E. 1983. Linguistic variation in Peruvian Spanish: unstressed vowel and / s /. Tesis doctoral: Universidad de Minesota.

Jakobson, R. y M. Halle. 1956. Fundamentals of language. La Haya: Mouton.

Keating. 1988. A survey of phonological features. Indiana University Linguistics Club. Bloomington.

1991. "Coronal places of articulation". En: Paradis y Prunet (eds.).

Kiparsky, P. 1985. "Some consequences of lexical phonology". Phonology Yearbook. 2: 85-138.

Ladefoged, P. 1982. A course in Phonetics. London: Harcourt Brace Jovanovich.

Ladefoged, P. y I. Maddieson. 1986. "Some of the sounds of the world's languages". UCLA. Working papers in phonetics. 64: 1-137.

Lipsky, J. 1983. "Reducción de / s / en el español de Honduras". Nueva Revista de Filología Hispánica. 32: 272-88.

1987. Fonética y fonología del español de Honduras. Tegucigalpa: Guaymuras.

López Chaves, J. 1977. "El fonema / s / en el habla de La Cruz, Sinaloa". Nueva Revista de Filología Hispánica. 26: 109-20.

López Morales, H. 1983. Estratificación social del español de San Juan de Puerto Rico. México: UNAM.

1989. Sociolingüística. Madrid: Gredos.

Maddieson, I. 1984. Patterns of Sounds. Cambridge: Cambridge University Press.

Montes Giraldo, J. J. 1984. "Algunos casos de / s / sonora en Colombia y sus implicaciones dialectales”. Homenaje a Luis Flórez. 220-7. Bogotá: Instituto Caro y Cuervo.

Navarro Tomás, T. 1952. Manual de pronunciación española. Madrid: Consejo Superior de Investigaciones Científicas.

Núñez Cedeño, R. et al. 1986. Estudios sobre la fonología del español del Caribe. Caracas: Ediciones La Casa de Bello. 
Paradis, C. y J. F. Prunet. 1989. “On coronal transparency”. Phonology . 6. 2: 317-48.

1991. Phonetics and phonology. The special status of coronals: internal and external evidence. San Diego \& London: Academic Press.

Poplack, S. 1979. Function and process in a variable phonology. Tesis doctoral. Filadelfia: Universidad de Pennsylvania.

Resnick, M. 1975. Phonological variants and dialect identification in Latin American Spanish. Nueva York: Universidad de Rochester.

Sanicky, C. 1982/83. "Las manifestaciones de la 's' final en el habla de Misiones". Boletín de Filología. Santiago.

Terrel, T. 1978a. "Constraints on the aspiration and deletion of final / s / in Cuban and Puerto Rican Spanish". The Bilingual Review. 4: 325-6.

1978b. "Sobre la aspiración y elisión de la / s / implosiva y final en el español de Puerto Rico". Nueva Revista de Filología Hispánica. 27: 24-38.

1979. "Final / s / in Cuban Spanish". Hispania. 62: 599-612.

Zamora Munné, J. C. y J. M. Guitarte. 1982. "Fonología dialectal hispanoamericana". Dialectología hispanoamericana: teoría-descripción-historia. Salamanca: Publicaciones del Colegio de España.

Zamora Vicente, A. 1970. Dialectología española. Madrid: Gredos. 